\title{
Endogenous Cycles and Human Capital
}

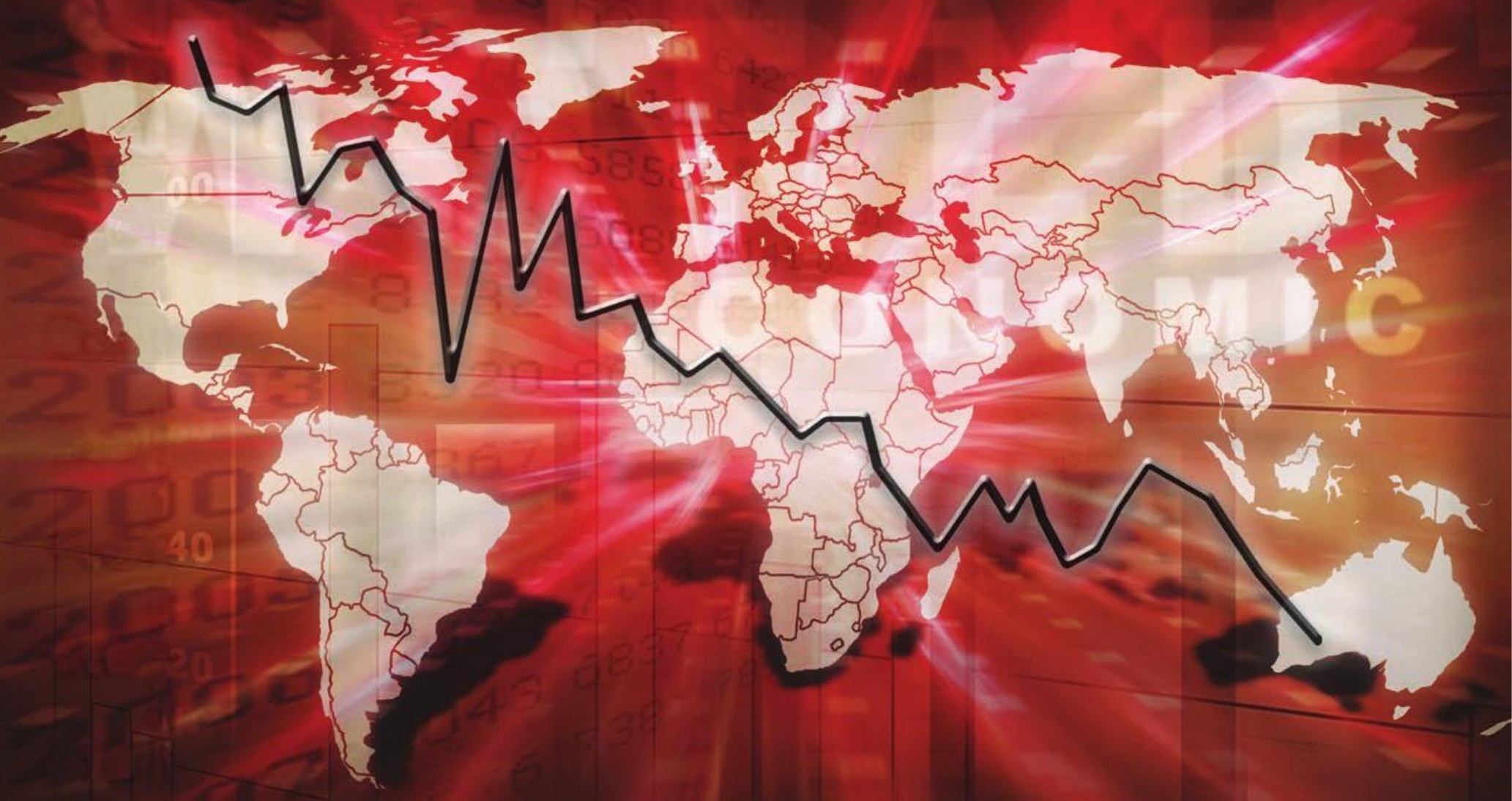

Dimitrios Varvarigos, University of Leicester

Working Paper No. 13/18

September 2013 


\title{
Endogenous Cycles and Human Capital
}

\author{
Dimitrios Varvarigos ${ }^{\ddagger}$ \\ University of Leicester
}

This Version: 26 July 2013

\begin{abstract}
Existing evidence shows that activities promoting the formation of human capital are countercyclical. In a two period OLG model, I show that countercyclical investment in human capital arises as a result of a parametric combination relating to preferences and technologies. This countercyclical reaction is responsible for the non-monotonicity in the evolution of human capital, thus initiating a self-sustained sequence of events that generate endogenous cycles, and possibly chaotic dynamics.
\end{abstract}

JEL classification: $\mathrm{E} 32, \mathrm{O} 41$

Keywords: Human capital, Cycles

* This is a heavily altered version of an existing paper, originally entitled "Fertility choice, human capital accumulation, and endogenous volatility" (Department of Economics working paper no. 11/08, University of Leicester). I would like to thank seminar participants at the University of Sheffield, the Athens University of Economics and Business, and the University of Cyprus for useful comments and suggestions.

‡ Address: Department of Economics, Astley Clarke Building, University Road, Leicester LE1 7RH, United Kingdom. Telephone: ++44 (0) 1162522184 Email: dv33@le.ac.uk 


\section{Introduction}

There can be little doubt on the importance of human capital for economic dynamics. Following the work of Lucas (1988), Azariadis and Drazen (1991), and Glomm and Ravikumar (1992), it has been an almost indispensable aspect of endogenous growth theories. Furthermore, empirical analyses have shown that human capital is a major contributor to economic growth (Benhabib and Spiegel 1994; Barro 2001). Of course, economic growth is not the only aspect of economic dynamics. Another indisputable fact is that major economic variables, such as per capita GDP, display cyclical patterns over time. Periods of thriving economic activity are followed by periods of anaemic economic conditions and vice versa. Given this, it is natural to ask whether there are any human capitalbased implications on the analysis of economic cycles.

Existing studies offer an affirmative reply. Particularly, a considerable body of literature has shown that activities that broadly constitute investment in human capital appear to be countercyclical. Betts and McFarland (1995) examine full-time attendance in community colleges in the Unites States and find that it is negatively correlated to the rate of unemployment. Sakellaris and Spilimbergo (2000) show that the enrolment of students from OECD countries in US universities, is inversely related to the phase of their home countries' economic cycle. DeJong and Ingram (2001) estimate a real business cycle model with human capital accumulation. Using measurable US data on consumption, investment and labour hours, in order to infer movements in skill-acquisition activities, they find that human capital investment is negatively correlated to output movements. Dellas and Sakellaris (2003) use college enrolment data from the Unites States and find that the propensity to enrol is negatively associated with macroeconomic aggregates such as GNP. Countercyclical enrolment patterns are also identified in the empirical investigations of Bedard and Herman (2008) and Johnson (2013) - the former for the case of male doctoral students; the latter for the case of females that enrol to graduate school.

The argument that is put forward to elucidate the distinctively countercyclical nature of human capital investment is at the same time indicative of the causal effect at work: according to conventional wisdom, periods of weak economic activity are exactly those periods where the opportunity cost of devoting time or effort towards the improvement of human capital, rather than earning labour income, is low. Thus, according to this argument, the movements of output above or below its trend represent the generating cause of 
variations in activities that promote human capital, such as formal education, skill acquisition etc. This paper takes the view that the dynamic implications from this argument are far more pervasive to what has hitherto been assumed. By inducing the reduction of human capital investment, a current increase of output may also cause a reduction in future labour productivity - in essence, it places the foundations for a future decrease in output. The distinction between cause and effect becomes blurred as activities that promote human capital do not just respond passively to the phase of the economic cycle. Instead, their response may act as a propagation mechanism that intensifies the non-monotonicity of economic dynamics, hence perpetuating the incidence of economic cycles.

A similar argument has already been identified in a seminal analysis by Kaas and Zink (2007). In their model, innovation and human capital are the driving forces behind productivity improvements. However, advanced technologies are skill-biased in the sense that they require technology-specific skills that are more costly to obtain. Therefore, productivity growth increases the cost of education, thus reducing the fraction of agents who invest in human capital improvements. This structure generates feedbacks that ultimately lead to endogenous growth cycles.

The purpose of the current paper is to provide a different framework that identifies conditions under which countercyclical human capital investment can be crucial in generating the incidence of economic cycles endogenously. I build a two period overlapping generations model in which agents use efficient labour - that is, raw labour time augmented by the stock of human capital - in order to produce output. Investment in human capital requires time/effort by the young, and an intergenerational externality whereby the current human capital stock complements private resources in supporting its accumulation. As long as some parameter conditions are jointly met, then the optimal amount of time devoted to human capital investment is decreasing in the current stock of human capital. Given that output is positively related to the human capital stock, the previous mechanism reveals that human capital investment is countercyclical. The parameter conditions under which this result may emerge are (i) an elasticity of intertemporal substitution that is above unity ${ }^{1}$, and (ii) diminishing returns in the accumulation of human capital in the sense that, holding the time devoted to human capital promoting activities constant, the accumulation of human

\footnotetext{
${ }^{1}$ Mulligan (2002), Gruber (2006) and Kapoor and Ravi (2010) provide empirical estimates that support an elasticity of intertemporal substitution above unity.
} 
capital is increasing but concave to the intergenerational externality that is manifested through the direct effect of the current human capital stock. ${ }^{2}$ When these conditions are jointly satisfied, the dynamics of human capital can become non-monotonic, meaning that rather than converging to a stationary equilibrium, the economy may admit a periodic equilibrium, converging to stable limit cycles during which human capital and output fluctuate permanently around their fixed points. What is particularly interesting is that, when this happens, the countercyclical nature of human capital-promoting activities is more than a mere response to economic cycles; it is at the same time the foundation of the complex economic dynamics that help generate and perpetuate cycles in the first place.

The preceding discussion reveals that my framework belongs to the class of models in which economic cycles are deterministic and emerge as a result of conditions linked to the deep structure of the economy, rather than the presence of exogenous shocks. ${ }^{3}$ While there is a significant number of papers that show the emergence of limit cycles under a variety of settings, the results of my analysis are novel enough to offer new insights that complement the existing literature by enriching our current understanding of the conditions under which cyclical dynamics may emerge. Firstly, my model explains a recognised stylised fact (i.e., the countercyclical nature of human capital investment) in terms of a well-defined combination of parameter values that involves both intertemporal substitution and the relative strength of the intergenerational externality in the evolution of human capital. Secondly, for reasons that were outlined in the previous paragraph, the same combination is ultimately responsible for the existence of cyclical trajectories. To the best of my knowledge, my model is the first to identify this parametric combination as the ultimate source of complex dynamics in a model with human capital. In fact, what is striking is that complex dynamics can emerge in a very simple and tractable model in which there are no elements that some previous analyses have employed in order to generate deterministic cycles in dynamic macroeconomic models. Such elements include, among others, consumption externalities (e.g., Lahiri and Puhakka 1998; Bunzel 2006); productive externalities (e.g., Benhabib and Farmer 1994; Azariadis and Reichlin 1996); public policy (e.g., Farmer 1986; Bhattacharya and Qiao 2007; Fanti and Gori 2011); money (e.g., Grandmond 1985; Michener and Ravikumar 1998); environmental

${ }^{2}$ Note that in Benhabib and Farmer (1994) and Azariadis and Reichlin (1996), endogenous cycles necessitate the existence of increasing returns in the accumulation of the reproducible factor of production, which is physical capital in these models.

${ }^{3}$ See Reichlin (1997) and the references therein. 
quality (e.g., Seegmuller and Verchère 2004; Palivos and Varvarigos 2010) or financial intermediation (e.g., Banerji et al. 2004). Furthermore, it should be noted that whereas existing analyses have focused on intertemporal substitution as an important element in the determination of complex dynamics in production economies (e.g., Reichlin 1986), they have done so in models where the reproducible factor of production is physical capital. Although both physical and human capital can act as the means of transferring real resources intertemporally, they differ by their very nature and by the mechanisms that govern their dynamics. In terms of my model, the focus on particular characteristics that govern the evolution of human capital - such as time spent on learning activities and intergenerational externalities - are of paramount importance for the results; thus, the distinction between physical and human capital is not a trivial one.

The structure of the paper is as follows. In Section 2, I outline the economic environment. Section 3 derives the temporary equilibrium while Section 4 analyses the dynamic equilibrium and shows the existence of limit cycles. In Section 5, I show that the framework may exhibit period doubling bifurcations that lead to chaotic dynamics. Section 6 concludes.

\section{The Economy}

Consider an economy in which time takes the form of discrete periods that are indexed by $t$. The economy is populated by overlapping generations of agents/households who face a lifespan of two periods - youth and old age. The population mass of each age cohort is normalised to 1 . The different generations of agents are connected by an intergenerational externality that equips a young agent with the average stock of human capital, denoted $\bar{h}_{t}$, available at the beginning of period $t{ }^{4}$

At the beginning of her youth, the individual is also endowed with a unit of time and a technology that allows her to transform efficient labour (i.e., raw time augmented by the stock of knowledge and expertise) into units of the economy's homogeneous good. During her youth, she also decides how much to consume and how much effort to devote for the

\footnotetext{
${ }^{4}$ Alternatively, I could have assumed that households begin their adulthood with only a fraction, say $\zeta \in(0,1]$, of the economy's average stock of human capital. This would only introduce a scale factor to the production function for young households as well to the evolution of human capital, without bearing on the model's qualitative results which would remain identical. For this reason, and to save on notation, I normalise $\zeta=1$.
} 
accumulation of human capital. Given that the economy's homogeneous good is perishable and non-storable, the accumulation of human capital is the only way she can transfer real resources towards her old age. When old, she combines her human capital together with a unit of time and produces units of output by utilising her technology. She decides how much to consume, and at the end of the period she passes away naturally.

For an agent born in period $t$, lifetime utility is given by

$$
u\left(c_{t}, d_{t+1}\right)=\frac{c_{t}^{1-\sigma}-1}{1-\sigma}+\beta \frac{d_{t+1}^{1-\sigma}-1}{1-\sigma}, \quad \beta \in(0,1), \sigma>0,
$$

where $c_{t}$ denotes consumption during youth and $d_{t+1}$ denotes consumption during old age. As it is evident from the utility function presented in Equation (1), the elasticity of intertemporal substitution, corresponding to $-\frac{d_{t+1} / c_{t}}{u_{d_{t+1}}(\bullet) / u_{c_{t}}(\bullet)} \frac{\partial\left(u_{d_{t+1}}(\bullet) / u_{c_{t}}(\bullet)\right)}{d_{t+1} / c_{t}}=\frac{1}{\sigma}$, may admit values that differ from one. This possibility will have major implications for the economy's equilibrium dynamics as we shall see later.

During her youth, the agent decides how to divide her time between the production of output and the accumulation of human capital. Therefore, her consumption during youth is determined by

$$
c_{t}=\left(1-e_{t}\right) \bar{h}_{t},
$$

where $e_{t}$ denotes the fraction of her time spent on such activities as formal education; skill acquisition; vocational training etc.; in short, any activity that augments an individual's knowledge, skills and expertise. Therefore, it effectively captures investment in human capital. This investment (taking the form of time/effort) is combined with the existing stock of human capital so as to generate the stock of human capital that will be available during her old age. Denoting the latter by $h_{t+1}$, it evolves according to

$$
h_{t+1}=\varphi e_{t} \bar{h}_{t}^{\psi}, \quad \varphi>0, \psi \in(0,1] .
$$

Note that the case where $\psi \neq 1$ implies that the technology determining the evolution human capital exhibits diminishing returns with respect to the existing capital stock. This is another crucial characteristic that will govern the equilibrium dynamics - an issue that will be formally discussed later in my analysis.

As it is evident from (3), the exponents on the variables that determine the formation of human capital are different. This is certainly not an alien assumption as it has appeared 
extensively in the existing literature (e.g., Glomm and Ravikumar 1992; de la Croix and Monfort 2000; Rillaers 2001). A possible criticism to this assumption relates to the idea that the use of effective time should be the main input in the formation of human capital, in exactly the same manner as in the production of output. Nevertheless, the specification in (3) is not inconsistent with this approach. For example, suppose that human capital formation is $h_{t+1}=\varphi\left(e_{t} \bar{h}_{t}\right)^{b} J\left(\bar{h}_{t}\right)$, where $e_{t} \bar{h}_{t}$ is education time in effective terms, $b>0$ and $J^{\prime}\left(\bar{b}_{t}\right)<0$, capturing the idea that, for given effort towards learning activities, a higher stock of human capital makes it more difficult to achieve genuine advancements in knowledge that will constitute an actual increase in human capital. Specifying $J\left(\bar{b}_{t}\right)=h_{t}^{-\lambda}$, such that $b>\lambda>0$, the specification in (3) emerges with the use of the composite term $\psi=b-\lambda$ and setting $b=1$ for simplicity.

When old, the agent combines the whole unit of time together with her stock of human capital in order to produce output. She uses the income received from this activity to satisfy her consumption needs when old. Hence, her consumption during old age is dictated by

$$
d_{t+1}=h_{t+1} .
$$

The previous analysis constitutes the analytical description of the economic environment. Thus, the model summarised through Equations (1)-(4) can be used to derive the economy's temporary and dynamic equilibrium and analyse their characteristics.

\section{The Temporary Equilibrium}

The temporary equilibrium can be described through

Definition 1. The temporary equilibrium of the economy is a set of quantities $\left\{c_{t}, d_{t+1}, e_{t}, b_{t+1}, \bar{h}_{t}\right\}$ such that:

(i) Given $\bar{h}_{t}$, the quantities $c_{t}, d_{t+1}, e_{t}$ and $h_{t+1}$ solve the optimisation problem of an agent born in $t$;

(ii) $\bar{b}_{t+j}=h_{t+j}$ for $j=0,1,2, \ldots$. 
It should be noted that the second part of Definition 1 holds due to the homogeneity of the population within an age cohort and the normalisation of its size to a unit mass. It is also straightforward to establish that the model generates interior equilibria for the variables that comprise the agent's set of choices. For this reason, we can substitute Equations (2)-(4) in (1) in order to express the problem as

$$
e_{t}=\arg \max \left\{\frac{\left[\left(1-e_{t}\right) h_{t}\right]^{1-\sigma}}{1-\sigma}+\beta \frac{\left(\varphi e_{t} h_{t}^{\psi}\right)^{1-\sigma}}{1-\sigma}\right\},
$$

where

$$
0<e_{t}<1 \text {. }
$$

After some straightforward algebra, we can find the solution for $e_{t}$ as

$$
e_{t}=\frac{\omega h_{t}^{\delta}}{1+\omega h_{t}^{\delta}}=\varepsilon\left(h_{t}\right),
$$

where

$$
\omega=\left(\beta \varphi^{1-\sigma}\right)^{\frac{1}{\sigma}} \text { and } \delta=\frac{1-\sigma}{\sigma}(\psi-1) .
$$

It is also instructive to write down the solution for the time that the agent devotes for the production of output during her youth. Denoting this by $l_{t} \equiv 1-e_{t}$, we can use the result in Equation (6) to write

$$
l_{t}=1-\varepsilon\left(b_{t}\right)=\lambda\left(b_{t}\right) .
$$

The solutions given in Equations (6) and (8) allow us to clarify a previous remark on the importance of the parameters $\sigma$ and $\psi$. In order to formalise the argument, let us begin by setting $\sigma=1$. In this case, the composite parameter terms in (7) become

$$
\omega=\beta \text { and } \delta=0 .
$$

Therefore, the solutions in (6) and (8) are reduced to

$$
e_{t}=\frac{\beta}{1+\beta}=\bar{e},
$$

and

$$
l_{t}=\frac{1}{1+\beta}=\bar{l} .
$$

It is obvious that, as long as $\sigma=1$, the optimal allocation of time during youth is invariant to the existing stock of human capital. The reason for this outcome is as follows. 
The stock of human capital generates substitution and income effects through its presence in the technology that determines the young adult's output (see Equation 2) and in the technology that determines the accumulation of human capital (see Equation 3) which, in turn, dictates the amount of output at the disposal of the agent during her old age (see Equation 4). If the elasticity of intertemporal substitution is restricted to be equal to one, then the magnitude of these effects is such that they cancel each other out.

Nevertheless, the elasticity of intertemporal substitution is not the only important factor in the determination of an agent's optimal allocation of time. As mentioned previously, the parameter $\psi$ is also crucial in this respect. To see this, let us assume that the dynamics of human capital involve constant returns, i.e., $\psi=1$. Using (7), we can see that $\delta=0$. Therefore,

$$
e_{t}=\frac{\left(\beta \varphi^{1-\sigma}\right)^{\frac{1}{\sigma}}}{1+\left(\beta \varphi^{1-\sigma}\right)^{\frac{1}{\sigma}}}=\breve{e},
$$

and

$$
l_{t}=\frac{1}{1+\left(\beta \varphi^{1-\sigma}\right)^{\frac{1}{\sigma}}}=\breve{l},
$$

are time invariant, for any $\sigma>0$. The existing stock of human capital does not generate any changes in the intertemporal consumption profile, simply because its effect on consumption is symmetric across periods. It is only when this effect is asymmetric, due to diminishing returns in the evolution of human capital, that the elasticity of intertemporal substitution generates the effects that are summarised in

Proposition 1. Consider $\sigma \neq 1$ and $\psi \in(0,1)$. The optimal allocation of time is summarised as follows:

(i) If $\sigma \in(0,1)$ then $\varepsilon^{\prime}\left(h_{t}\right)<0, \lambda^{\prime}\left(h_{t}\right)>0$;

(ii) If $\sigma>1$ then $\varepsilon^{\prime}\left(h_{t}\right)>0, \lambda^{\prime}\left(h_{t}\right)<0$.

Proof. Substitute (7) in (6) and use the resulting expression in (8). Subsequently, calculate the derivatives of $\varepsilon\left(h_{t}\right)$ and $\lambda\left(h_{t}\right)$ with respect to $h_{t}$. 
Let us try to understand the intuition behind these results by considering the impact of a higher human capital stock. Naturally, this implies that the utility cost of devoting time to activities that increase human capital (i.e., the opportunity cost of not producing output during youth) is higher. This is because the higher stock of human capital increases the amount of income received (and, correspondingly, the amount of goods that can be purchased and consumed) for every unit of labour time devoted during the person's youth. The substitution effect induces the agent to increase labour time at the expense of the time she spends accumulating human capital. Nevertheless, there is an income effect as well. Given the convexity of preferences, the agent will optimally wish to smooth her consumption profile over the lifetime. The only way she can achieve this is by accumulating human capital - doing so will increase the resources that she can produce during her old age, and therefore allow her to consume more during this later stage of her lifespan. This effect will induce her to reduce the time she spends producing output when young.

There is a second set of substitution and income effects, however. These effects relate to the impact of the current human capital stock on the future formation of human capital consequently, the amount of income received during old age, and therefore the utility benefit from old age consumption. The substitution effect will induce the agent to spend more time accumulating human capital during her youth, at the expense of the time she spends producing output. However, there is an income effect related to the fact that the agent wants to smooth her consumption profile. In this case, she can achieve this by increasing her income, and therefore consumption during youth. Optimally, she will reduce the time spent on the accumulation of human capital and she will increase the time she devotes to the production of output. The elasticity of intertemporal substitution determines whether income or substitution effects dominate. Given the previous discussion, however, we have two sets of such effects that work in the opposite direction. So what determines the ultimate outcome? As summarised in Proposition 1, for given values of $\sigma$, the ultimate outcome will be determined by the presence of diminishing returns in the accumulation of human capital, i.e., $\psi<1$. We can clarify the intuition as follows. Suppose that $\sigma \in(0,1)$ so that the substitution effects dominate the income effects. For $\psi<1$ and an increase in the stock of human capital, the utility benefit from old age consumption is weaker compared to the utility benefit from the consumption during young adulthood. Correspondingly, the agent will find optimal to decrease the time she spends accumulating human capital and at the same time, 
increase her effort towards production. Now, suppose that $\sigma>1$ so that the income effects dominate the substitution effects. For $\psi<1$ and a higher stock of human capital, the income effect will dominate as the agent strives to smooth her intertemporal consumption profile. As a result, the young person will find optimal to increase the time she spends accumulating human capital. Furthermore, she will optimally reduce her effort towards the production of output when young.

The qualitative nature of these effects reveals that they may have significant repercussions for the dynamics of human capital accumulation. These issues are formally analysed and discussed in the subsequent section.

\section{The Dynamic Equilibrium}

We can start by substituting $\bar{h}_{t}=h_{t}$ together with (6) in Equation (3) to get

$$
h_{t+1}=\frac{\varphi \omega h_{t}^{\psi+\delta}}{1+\omega h_{t}^{\delta}}=F\left(h_{t}\right) .
$$

This first order difference equation describes the dynamics of human capital accumulation. Thus, the economy's dynamic equilibrium is described in

Definition 2. The dynamic equilibrium is a sequence of temporary equilibria that satisfy $h_{t+1}=F\left(h_{t}\right)$.

Earlier, we identified the fact that the elasticity of intertemporal substitution is a potential source of different effects on the determination of the optimal time allocation. For this reason, it is instructive to examine the dynamic outcomes that transpire for different configurations of its value. Given that such outcomes require diminishing returns to the accumulation of human capital, the remaining analysis will make use of $\psi \neq 1$ so that the parameter $\psi$ is restricted on the interval $(0,1)$. Furthermore, the subsequent analysis will be applying the notation $\hat{b}$ to indicate an interior solution satisfying $\hat{b}=F(\hat{b})$. Given these, I will begin the formal characterisation of the dynamic equilibrium with

Proposition 2. If $\sigma \geq 1$ there exists a unique asymptotically stable steady state $\hat{b}>0$. For $h_{0}>0$, such that $h_{0} \neq \hat{b}$, the dynamics will converge monotonically towards $\hat{b}$. 
Proof. See the Appendix.

The dynamics associated with the previous proposition are illustrated in Figure 1. Let us now turn to the intuition behind the result of Proposition 2. When $\sigma=1$, the optimal effort towards activities that improve human capital is invariant to its current stock. Therefore, the stock of human capital will converge to a stationary equilibrium, while the dynamics of convergence are positively monotonic given $F^{\prime}\left(h_{t}\right)>0$ for $\psi \in(0,1)$. When $\sigma>1$, we have seen that the optimal time spend on activities that promote human capital is increasing in $b_{t}$ (see Proposition 1ii). Therefore, there is a complementarity between the existing human capital stock and human capital investment. Despite the fact that this complementarity could place the foundations for multiple equilibria, in this case it is not strong enough to do so. This is because the return to human capital investment is still high when the existing human capital stock is relatively low. Consequently, the rate of human capital formation guarantees a unique interior equilibrium to which the economy converges monotonically.

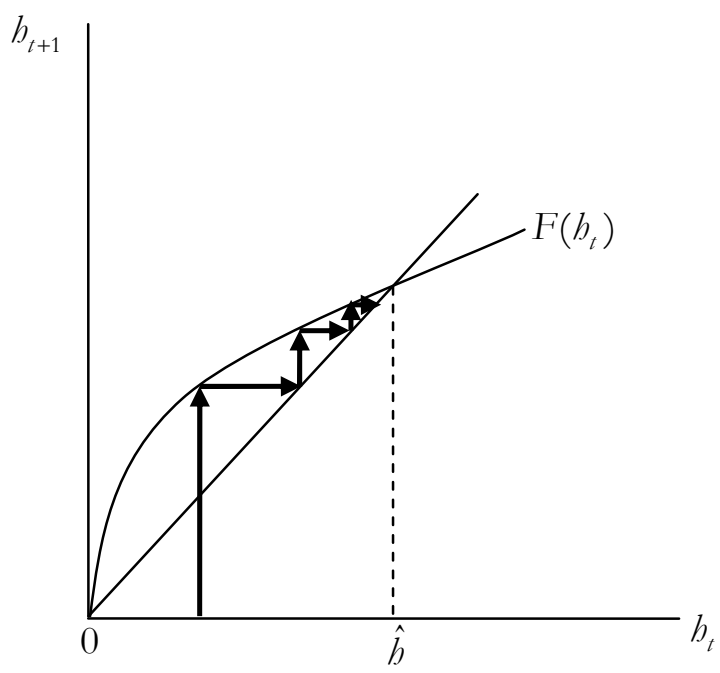

Figure 1. Monotonic dynamics for $\sigma \geq 1$

More interesting cases appear when $\sigma \in(0,1)$, i.e., when the outcomes that characterise the optimal time allocation are summarised in Proposition 1i. Now, the current stock of human capital has two conflicting effects on the determination of $h_{t+1}$. On the one hand, 
there is a positive effect from the intergenerational externality that is governed by the parameter $\psi$; on the other hand, there is a negative effect that emerges from the adverse response of human capital promoting activities to a higher human capital stock, i.e., $\varepsilon^{\prime}\left(b_{t}\right)<0$. These conflicting effects are suggestive of the possibility that the dynamics of human capital may be non-monotonic. As we shall see later, this possibility does materialise. However, this is not the case for every permissible value in the range $\sigma \in(0,1)$, as it is evident from

Proposition 3. If $\sigma \in(0,1)$ and $\sigma+\psi>1$, there exists a unique asymptotically stable steady state $\hat{b}>0$. For $h_{0}>0$, such that $h_{0} \neq \hat{b}$, the dynamics will converge monotonically towards $\hat{b}$.

Proof. See the Appendix.

When $\sigma+\psi>1$ the fact that $\varepsilon^{\prime}\left(h_{t}\right)<0$ is not sufficient to alter the shape of human capital dynamics; these are still monotonic. Things may change however when $\sigma+\psi<1$. In order to characterise the dynamics in this case, I will define the composite term

$$
\xi=\left[\frac{\omega \psi}{-(\delta+\psi)}\right]^{-\frac{1}{\delta}} .
$$

Note that $\xi>0$ because, by virtue of Equation (7), it is $\sigma+\psi<1 \Leftrightarrow \delta+\psi<0$. The equilibrium dynamics are described in

Proposition 4. If $\sigma \in(0,1)$ and $\sigma+\psi<1$, there exists a unique $\hat{b}>0$ such that:

(i) If $\xi>F(\xi)$ then $F^{\prime}(\hat{b}) \in(0,1)$. Therefore $\hat{b}$ is asymptotically stable and for $h_{0}>0$, such that $h_{0} \neq \hat{b}$, the dynamics will converge monotonically towards $\hat{b}$.

(ii) If $\xi<F(\xi)$ then $F^{\prime}(\hat{b})<0$. When $F^{\prime}(\hat{b}) \in(-1,0)$ bolds, then $\hat{b}$ is asymptotically stable and for $h_{0}>0$, such that $h_{0} \neq \hat{b}$, the dynamics will converge towards $\hat{b}$ through damped oscillations. When $F^{\prime}(\hat{b})<-1$ bolds, then $\hat{b}$ is unstable and oscillations in the dynamics of 
buman capital are permanent in the sense that there exists a limit cycle of period-2 and possibly limit cycles of period- $p$, where $p>2$.

Proof. See the Appendix.

The interesting dynamics are described in the second part of Proposition 4 and illustrated in Figures 2 and 3. As it is shown in the Appendix and illustrated in Figure 4, a period-2 cycle corresponds to the two additional fixed points (i.e., those in addition to 0 and $\hat{b}$ ) of the second iterate of the function in (14), that is $F^{2}\left(b_{t}\right)$.

What is the intuition behind the occurrence of cyclical trajectories? At relatively high values for the human capital stock, the slope of the transition equation in (14) may actually change sign and become negative. If the fixed point generated by (14) lies on the downward sloping part of the transition graph, convergence to the steady state may be cyclical rather than monotonic. There may even be convergence to a stable cycle - human capital may fluctuate permanently around its fixed point. The intuition for this result is the following. Suppose that the human capital stock is low. Young adults will respond by devoting more time to the accumulation of human capital and less time producing output. Next period however, the available stock of human capital will be relatively high as a result of the previous generation's effort. This will induce young adults to produce more output at the expense of the time they spend accumulating human capital. The latter effect implies a lower endowment of human capital for the subsequent generation of young adults. As a result, they will decide to invest more time to the accumulation of human capital, at the expense of output production, and so on. 


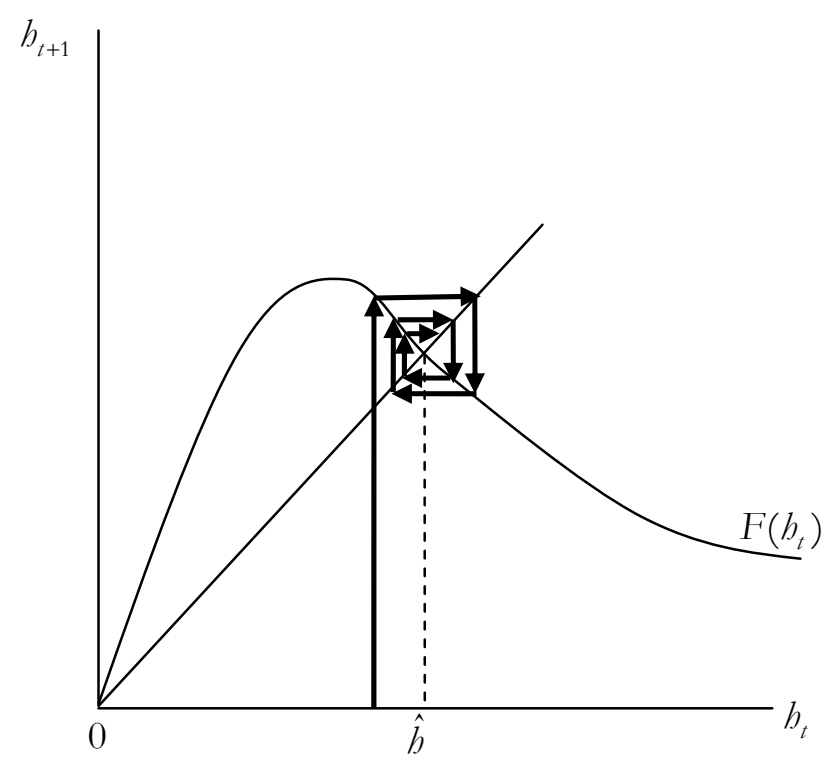

Figure 2. Damped oscillations with $\sigma \in(0,1)$

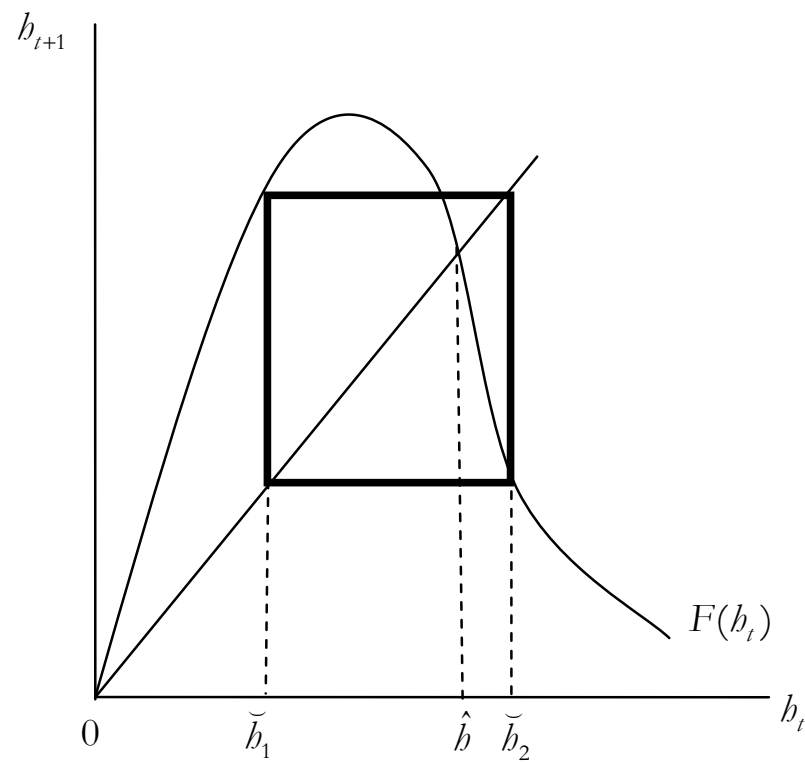

Figure 3. A period-2 cycle with $\sigma \in(0,1)$ 


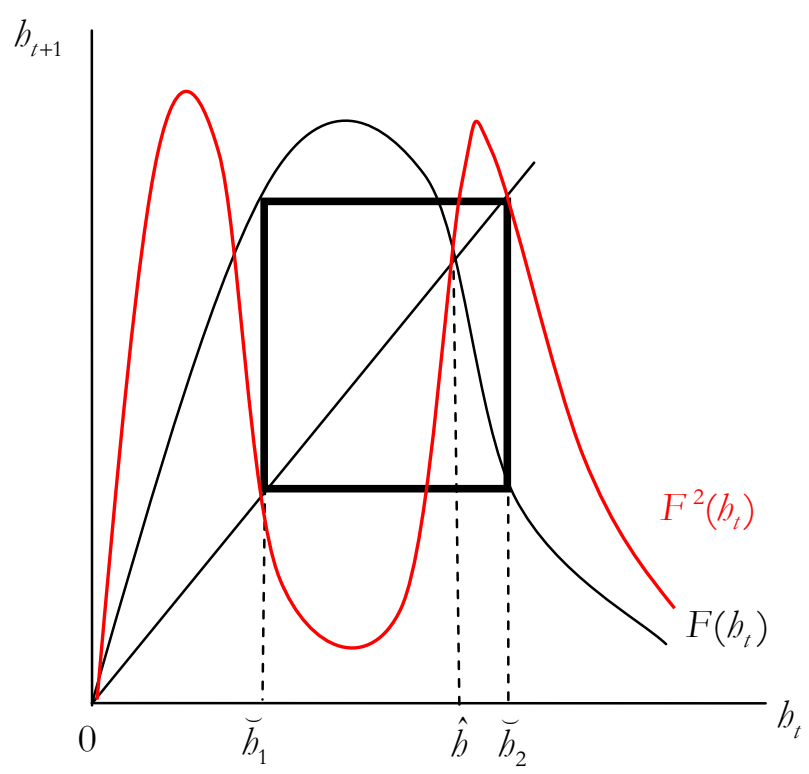

Figure 4. The period-2 cycle as the fixed points of $F^{2}\left(b_{t}\right)$

\subsection{Countercyclical Investment in Human Capital}

The preceding discussion allows us to relate the incidence of cyclical fluctuations with the cyclical nature of activities that constitute investment in human capital, such as effort on formal education or any other learning activity captured by the variable $e_{t}$ in my model. This can be illustrated as follows. In every period there are two cohorts of agents producing output - the young and the old. Given the model's assumptions, each young person earns income according to

$$
y_{\text {joung }}\left(b_{t}\right)=\lambda\left(b_{t}\right) h_{t},
$$

whereas each old person's income is equal to

$$
y_{\text {old }}\left(h_{t}\right)=h_{t} .
$$

The result in Proposition 1 reveals that, as long as parameter values are conducive to the emergence of endogenous volatility, it is $\lambda^{\prime}\left(h_{t}\right)>0$. Therefore, we have $y_{\text {young }}^{\prime}\left(h_{t}\right)>0$ and $y_{\text {old }}^{\prime}\left(h_{t}\right)>0$. Given that each cohort has a population mass of 1 , income per capita (denoted $i_{t}$ ) is given by 


$$
i_{t}=\frac{y_{\text {young }}\left(h_{t}\right)+y_{\text {old }}\left(h_{t}\right)}{2} \equiv \iota\left(h_{t}\right)
$$

so that $\iota^{\prime}\left(h_{t}\right)>0$.

Now, consider some period $T$ and suppose that the model generates endogenous cycles through periodic equilibria - say a period-2 cycle $\left\{\breve{h}_{1}, \breve{h}_{2}\right\}$ such that $\breve{h}_{1}<\hat{b}<\breve{h}_{2}{ }^{5}$ It is straightforward to use $\iota^{\prime}\left(h_{t}\right)>0$ and Proposition 1 to establish

$$
\varepsilon\left(h_{T}\right)-\varepsilon(\hat{b})\left\{\begin{array}{lll}
>0 & \text { when } & \iota\left(h_{T}\right)-\iota(\hat{b})<0 \\
<0 & \text { when } & \iota\left(h_{T}\right)-\iota(\hat{b})>0
\end{array} .\right.
$$

The main implication from the expression in (19) can be summarised in

Corollary 1. When the economy displays endogenous cycles, then activities that promote human capital are countercyclical in the sense that $\varepsilon\left(h_{t}\right)$ is above (below) its trend as long as income per capita is below (above) its trend.

The implication that is outlined in Corollary 1 is not just a mere theoretical curio. As I indicated in the introductory part of the paper, there exists a plethora of quantitative analyses that present clear evidence on the countercyclical nature of activities that promote human capital. The conventional approach to explaining this outcome is based on the 'opportunity cost' argument. In periods of booming economic activity, the opportunity cost of not devoting time or effort in order to earn labour income is high, whereas recessions are exactly the periods where this cost is low enough so that, coupled with the expectation of an improvement in future economic conditions, increases the incentive to forego current income in order to pursue activities that will increase future productivity. Such an approach clearly identifies a causal link from the state of the economy to the optimal decisions governing investment in human capital.

In my model, this effect is summarised in Corollary 1 but the combination of the results in Proposition 1i and Proposition 4ii allows me to explain this outcome in terms of a well-

\footnotetext{
${ }^{5}$ See the Appendix on why a period-2 cycle satisfies $\breve{b}_{1}<\hat{b}<\breve{b}_{2}$.
} 
defined parameter combination. Furthermore, in my model this outcome is just one side of the story. This is because the optimal response of $\varepsilon\left(h_{t}\right)$ is by itself the reason behind the non-monotonicity of the transition equation $h_{t+1}=F\left(h_{t}\right)$ that is ultimately responsible for the onset of endogenous cycles. For this reason, cause and effect are almost indistinguishable as the optimal investment in human capital feeds back to the economy's dynamics which subsequently strengthen the countercyclical nature of investment in human capital and so on, thus initiating a self-sustaining sequence of events that generate cyclical fluctuations endogenously.

\subsection{Introducing Long-Run Growth}

As it became evident from the preceding analysis, one of the necessary conditions for the existence of cyclical trajectories is $\psi \neq 1$. Indeed, when $\psi=1$ the solutions in (7) and (12) imply that the dynamics of human capital take the form $h_{t+1}=\varphi[\omega /(1+\omega)] h_{t}$. Therefore, in this case we do not observe any transitional dynamics at all. Instead, the economy lies always on a balanced growth path where long-run growth is positive as long as

$$
\frac{h_{t+1}}{h_{t}}-1=\frac{\varphi \omega}{1+\omega}-1>0 \text {. }
$$

Despite the previous argument, endogenous cycles in my model are not inconsistent with a situation of permanent growth in output. In order to clarify this issue, we should recall that the assumption of constant returns in the accumulation of the reproducible factor of production is not the only way to sustain an equilibrium with long-run growth. An alternative way is to introduce an additional process of productivity improvements. This is the scenario I am going to illustrate now.

Suppose that output production is augmented by a variable, denoted $A_{t}$, indicating total factor productivity (TFP). Furthermore, suppose that TFP grows at a constant rate $g>0$ so that

$$
\frac{A_{t+1}}{A_{t}}-1=g .
$$

Given this assumption, Equations (2) and (4) of the original problem are now written as

$$
c_{t}=A_{t}\left(1-e_{t}\right) \bar{b}_{t}
$$

and 


$$
d_{t+1}=A_{t+1} h_{t+1} .
$$

It is straightforward to check that, after solving the model under the new set-up suggested by Equations (21)-(23), the result for $e_{t}$ is the same as the one in (6). The only difference is the presence of a scale factor related to the growth component of TFP - a scale factor manifested in the composite term $\omega$ which now takes the form

$$
\omega=\left[\beta \varphi^{1-\sigma}(1+g)^{1-\sigma}\right]^{\frac{1}{\sigma}} .
$$

Consequently, the result in Proposition 4 remains intact in the sense that the same conditions can still generate endogenous cycles in human capital. The only difference is in terms of output. While in the original model, output movements are solely affected by the dynamics of human capital, in this case, the economy will experience growth cycles in output. In other words, the cyclical trajectories of human capital will generate output growth cycles around the equilibrium growth rate $g$.

\section{Bifurcations and Chaos}

With Proposition 4ii, I established the existence of a periodic equilibrium. The conditions necessary for such an equilibrium to exist can be summarised in

Corollary 2. Limit cycles may exist if and only if $\sigma+\psi<1$. Therefore, the following conditions must hold simultaneously: :

$$
\begin{aligned}
& \text { (i) } \frac{1}{\sigma}>1 \text {; } \\
& \text { (ii) } \quad \psi \neq 1 \text {. }
\end{aligned}
$$

As we can see, the elasticity of intetemporal substitution is not the only factor in generating endogenous cycles. In addition to this preferences factor, one of the technology parameters governing the dynamics of human capital is also a crucial characteristic in the emergence of a periodic equilibrium.

A striking aspect of this framework is that endogenous cycles can emerge from such a simple model. Despite this simplicity however, the only periodic solution that can be shown analytically is that of a period-2 cycle. Nevertheless, it is well-known that the dynamics of 
scalar systems that admit periodic equilibria may be sensitive to parameter changes that may cause flip - or period doubling - bifurcations. In other words, the period-2 cycle is probably not the only stable periodic equilibrium. Instead, different parameter configurations may lead to stable limit cycles of an order that is greater than 2. To illustrate such cases, I will undertake a numerical example. In particular, I set $\varphi=15, \beta=0.5, \psi=0.5$ and an initial value $h_{0}=40$. Then I vary the parameter $\sigma$ from 0.01 to 0.495 in order to guarantee that the conditions of Corollary 2 hold; that is, $\sigma+\psi<1$.

In Figure 5, we can see the bifurcation diagram from this simulation. It is evident that as $\sigma$ is reduced, the dynamic equilibrium changes. Specifically, as $\sigma$ approaches 0.15 , at some point the steady state equilibrium $\hat{b}$ becomes unstable and in its place we see the emergence of a stable period-2 cycle (see Figure 6). Somewhere close to 0.12 , the period-2 cycle becomes unstable and in its place we can see the emergence of a period-4 cycle (see Figure 7). This process of period doubling continues with cycles of period-8, period-16 and so on, as $\sigma$ takes progressively smaller values (see Figures 8 and 9). The dynamic behaviour becomes increasingly erratic until we see a window which illustrates the possibility of a period-3 cycle. From then on, further reductions of $\sigma$ are associated with a new process of period doubling that leads to cycles of period-6, period-12 and so on. The possible existence of a period-3 cycle in the previous numerical example is of high significance. By Sarkovskii's Theorem, a period-3 cycle implies the existence of limit cycles of any period, but also solutions which are aperiodic or chaotic ( $\mathrm{Li}$ and Yorke 1975). Such chaotic dynamics are illustrated in Figure 10. 

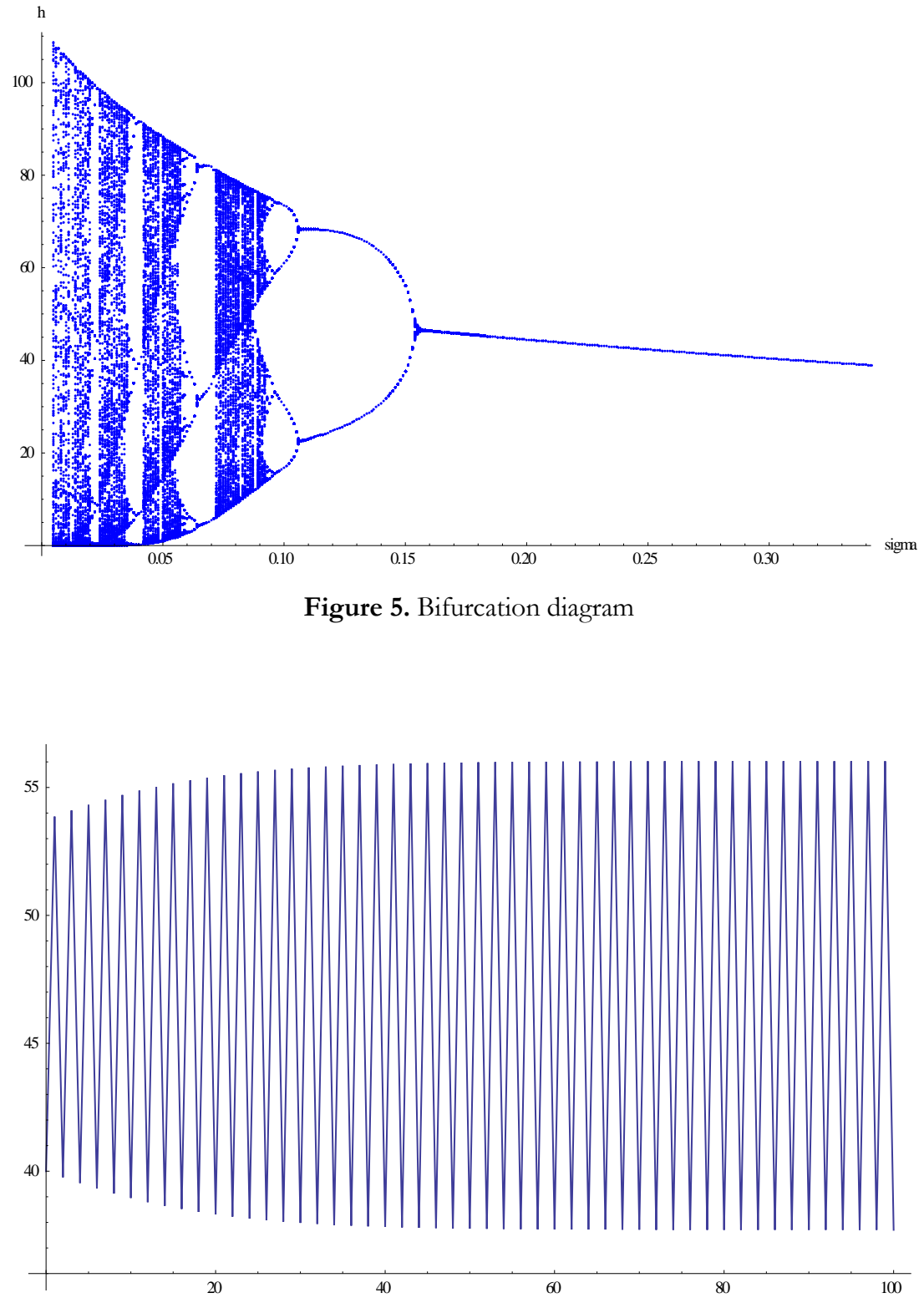

Figure 6. Period-2 cycle 


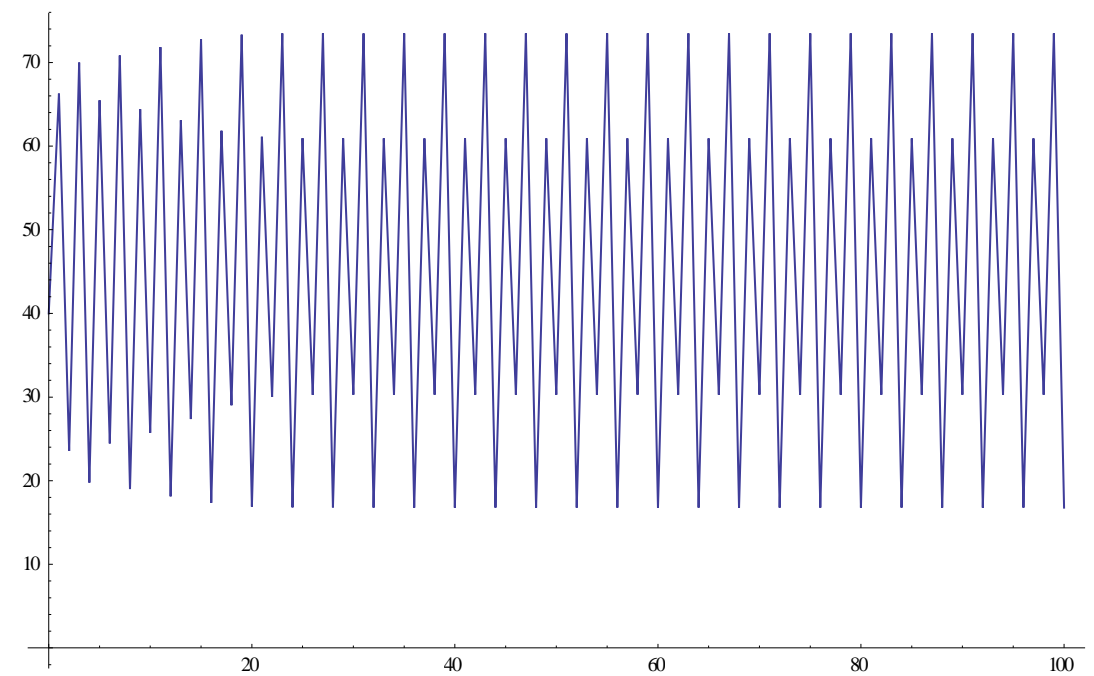

Figure 7. Period-4 cycle

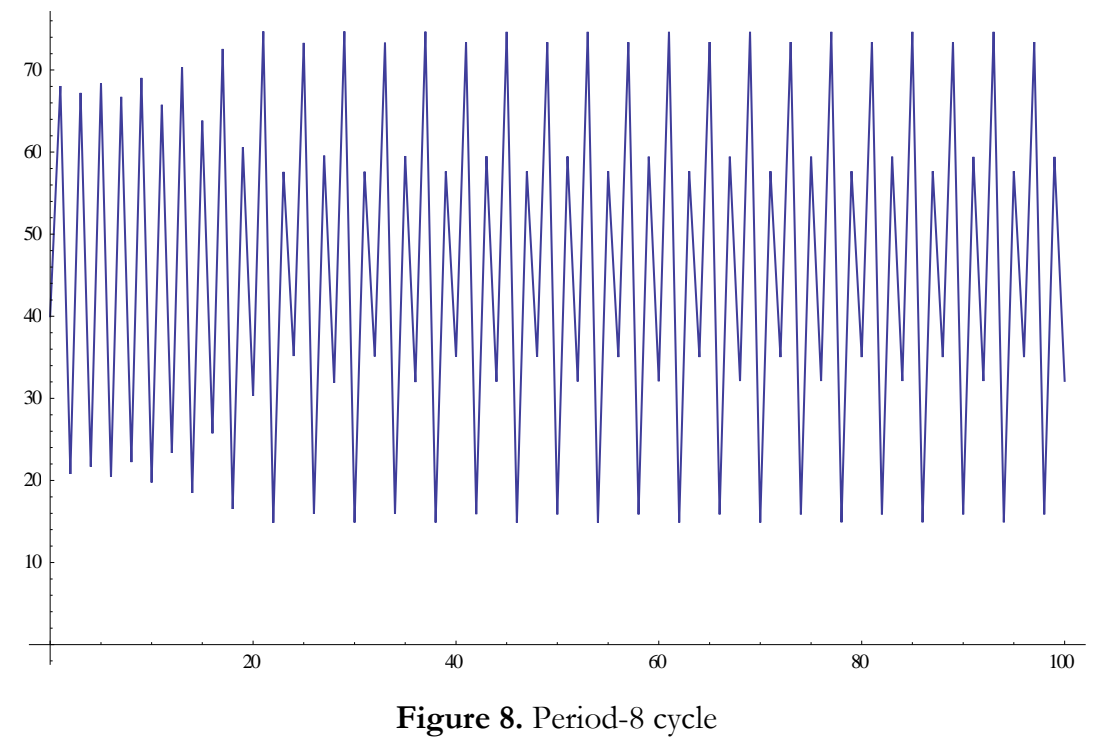




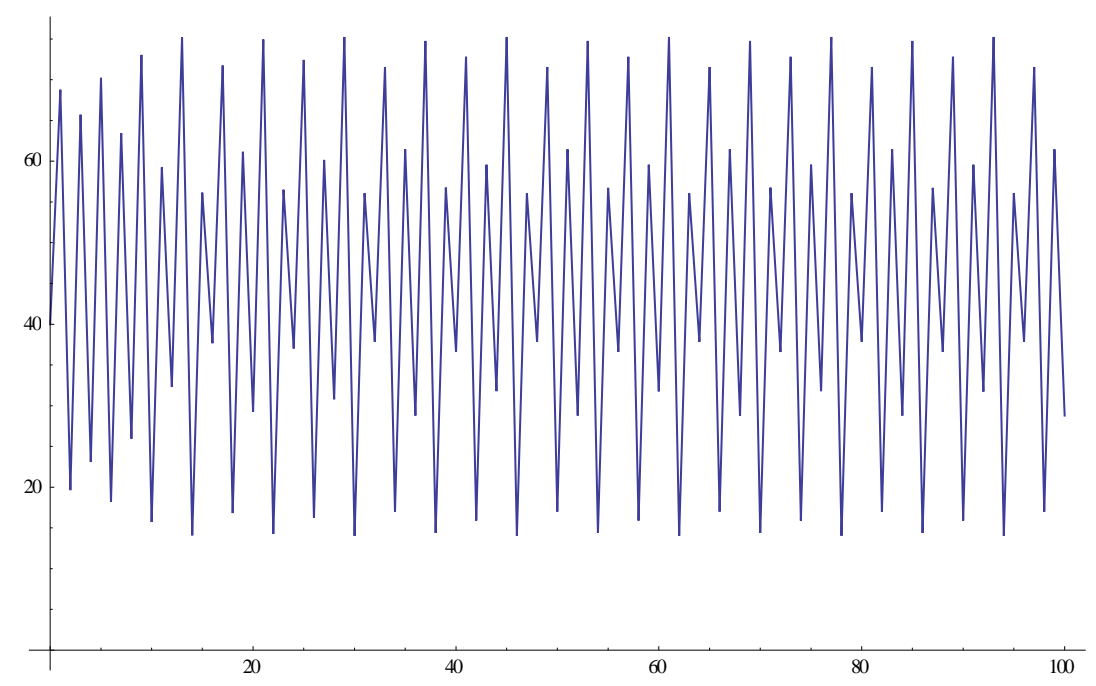

Figure 9. Period-16 cycle

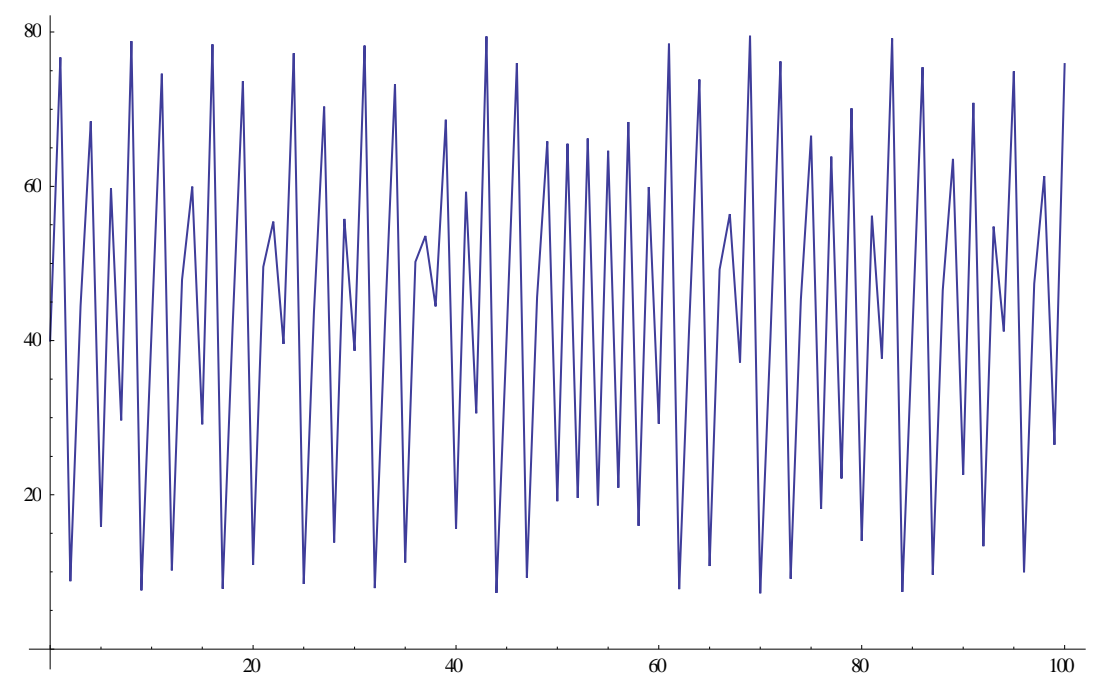

Figure 10. Aperiodic dynamics

\section{Conclusion}

Under what conditions is human capital investment countercyclical? Can the countercyclical nature of learning activities be a source of economic instability, given that human capital is a major factor of labour productivity? In this paper, my endeavour was to address these issues. Firstly, I identified a specific parametric combination under which learning activities are countercyclical. Secondly, under the same parameter condition, the dynamics may converge 
to limit cycles because the optimal response of human capital investment is ultimately responsible for non-monotonic dynamics. In fact, numerical examples suggest that these dynamics may even become chaotic.

The present framework reveals that the presence of human capital can generate cyclical trajectories and even erratic dynamics under a setting in which the more familiar overlapping generations (OLG) model with physical capital rules out such complex dynamics. Indeed, the standard OLG model with CRRA preferences, log-linear technologies and the absence of increasing returns results in a unique stationary equilibrium, when the reproducible factor of production is physical capital - see for example Proposition 9.4 (p. 334) in Acemoglu (2009). Nevertheless, the same conditions can be consistent with endogenous cycles in a model where human capital is the reproducible factor of production, as the present analysis has demonstrated. Given that the allocation of time is of the utmost importance when we consider human capital, this result echoes the seminal analysis of Reichlin (1986) in that the endogenous allocation of time can have significant repercussions for the dynamic behaviour of an otherwise typical OLG model. Moreover, it opens an important direction for further research since the incorporation of human capital in a variety of OLG settings could reveal that, under certain circumstances, economic instability may be a more common outcome to what our current understanding actually suggests.

\section{Appendix}

\section{A1 Proof of Proposition 2}

For the case where $\sigma=1$, we can substitute (6) and (9) in (14) to get

$$
h_{t+1}=\frac{\varphi \beta}{1+\beta} h_{t}^{\psi}=F\left(h_{t}\right) .
$$

We can see that there are two possible steady state equilibria. One is $h_{t+1}=h_{t}=0$ and the other is $h_{t+1}=h_{t}=\hat{b}=[\varphi \beta /(1+\beta)]^{1 /(1-\psi)}$. Given (A1.1), the derivative of $F\left(h_{t}\right)$ is

$$
F^{\prime}\left(h_{t}\right)=\frac{\psi \varphi \beta}{1+\beta} h_{t}^{\psi-1}
$$

It is straightforward to check that $F^{\prime}(0)=\infty$ and $F^{\prime}(\hat{b})=\psi \in(0,1)$. Thus, the only asymptotically stable equilibrium is $\hat{b}$. 
Now, let us consider what happens when $\sigma>1$. Note that in this case $\delta=\frac{\sigma-1}{\sigma}(1-\psi)>0$, it is $\delta+\psi=\left[\frac{\sigma-1}{\sigma}(1-\psi)+\psi\right] \in(0,1)$. Given these, it is $F(0)=0$ and $F(\infty)=\infty$. Furthermore, the derivative

$$
F^{\prime}\left(h_{t}\right)=\frac{\varphi \omega h_{t}^{\delta+\psi-1}}{1+\omega h_{t}^{\delta}}\left[\psi+\delta\left(1-\frac{\omega h_{t}^{\delta}}{1+\omega h_{t}^{\delta}}\right)\right],
$$

is positive, while $\mathrm{A}(1.3)$ reveals that $F^{\prime}(0)=\infty$, meaning that $h_{t+1}=h_{t}=0$ is an unstable solution. Next, we can define the function

$$
M\left(h_{t}\right)=\frac{F\left(h_{t}\right)}{h_{t}}=\frac{\varphi \omega h_{t}^{\delta+\psi-1}}{1+\omega h_{t}^{\delta}},
$$

That satisfies $M(0)=\infty$ and $M(\infty)=0$ for $0<\delta+\psi<1$. The derivative $M^{\prime}\left(b_{t}\right)$ is equal to

$$
M^{\prime}\left(h_{t}\right)=\frac{\varphi \omega h_{t}^{\delta+\psi-2}}{1+\omega h_{t}^{\delta}}\left[\delta\left(1-\frac{\omega h_{t}^{\delta}}{1+\omega h_{t}^{\delta}}\right)-(1-\psi)\right],
$$

and it is negative by virtue of $0<\delta+\psi<1$. We conclude that there is a unique interior $\hat{b}$ such that $h_{t+1}=h_{t}=\hat{b}$ and, therefore, $M(\hat{b})=1$. Furthermore, this solution satisfies $M^{\prime}(\hat{b})<0 \Leftrightarrow F^{\prime}(\hat{b})<1$. Combined with $F^{\prime}\left(h_{t}\right)>0$, this analysis reveals that $\hat{b}$ is asymptotically stable because $0<F^{\prime}(\hat{b})<1$.

\section{A2 Proof of Proposition 3}

With $\sigma \in(0,1)$ and $\sigma+\psi>1$, it is $\delta<0$ and $\delta+\psi>0$. From (14), these imply that $F(0)=0$ and $F(\infty)=\infty$. Now let us consider the derivative in (A1.3). Since $\delta<0$ and $\delta+\psi>0$, it is obvious that $0<\delta+\psi<1$ and that $F^{\prime}\left(h_{t}\right)>0$. Furthermore, it is $F^{\prime}(0)=\infty$, meaning that $h_{t+1}=h_{t}=0$ is an unstable solution. As for the function $M\left(b_{t}\right)$ in (A1.4), the same conditions reveal that $M(0)=\infty, M(\infty)=0$ and $M^{\prime}\left(h_{t}\right)<0$ still hold. Consequently, there is a unique interior $\hat{b}$ such that $h_{t+1}=h_{t}=\hat{b}$ and, therefore, $M(\hat{b})=1$. Furthermore, this solution satisfies $M^{\prime}(\hat{b})<0 \Leftrightarrow F^{\prime}(\hat{b})<1$. Combined with $F^{\prime}\left(b_{t}\right)>0$, it is clear that $\hat{b}$ is asymptotically stable because $0<F^{\prime}(\hat{b})<1$. 


\section{A3 Proof of Proposition 4}

When $\sigma \in(0,1)$ and $\sigma+\psi>1$, it is $\delta<0$ and $\delta+\psi<0$. Under these conditions, Equation (14) reveals that $F(0)=F(\infty)=0$. Furthermore, we can use Equation (A1.3) to establish that

$$
F^{\prime}\left(b_{t}\right)\left\{\begin{array}{lll}
>0 & \text { if } & b_{t}<\xi \\
<0 & \text { if } & h_{t}>\xi
\end{array}\right. \text {, }
$$

where $\xi>0$ is defined in Equation (15) of the main text. Additionally, we can check that (A3) reveals $F^{\prime}(0)=\infty$, therefore $h_{t+1}=h_{t}=0$ is an unstable solution.

Now, let us turn our attention to Equation (A4) and (A5). We can establish that $M(0)=\infty, M(\infty)=0$ and $M^{\prime}\left(b_{t}\right)<0$ still hold. These imply that there is a unique $\hat{b}$ such that $h_{t+1}=h_{t}=\hat{b}$ and, therefore, $M(\hat{b})=1$. Furthermore, it is $M^{\prime}(\hat{b})<0$ or, alternatively, $F^{\prime}(\hat{b})<1$. In this case, however, we cannot make any definite conclusions concerning the stability of this equilibrium as we do not yet know whether $\hat{b}$ lies on the downward sloping part of $F\left(h_{t}\right)$. For this reason, we have to examine two different scenarios.

Given the properties of the function $F\left(b_{t}\right)$, as long as $F(\xi)<\xi$ then $\hat{b}$ will lie on the upward sloping part of $F\left(b_{t}\right)$ and it will satisfy $\hat{b}<\xi$ and $0<F^{\prime}(\hat{b})<1$. In this case, $\hat{b}$ is asymptotically stable. When $F(\xi)>\xi, \hat{b}$ will lie on the downward sloping part of $F\left(b_{t}\right)$ and it will satisfy $\hat{b}>\xi$ and $F^{\prime}(\hat{b})<0$. This means that dynamics are oscillatory, rather than monotonic, and that there may be periodic solutions for which oscillations are permanent. To examine this formally, I will check the existence of a period-2 cycle by evaluating the function

$$
F^{2}\left(h_{t}\right)=F\left(F\left(h_{t}\right)\right)=\frac{\varphi \omega\left(F\left(h_{t}\right)\right)^{\psi+\delta}}{1+\omega\left(F\left(h_{t}\right)\right)^{\delta}}
$$

in order to check whether there are points $\breve{h}_{p} \neq 0$ and $\breve{h}_{p} \neq \hat{b}$ for which $F^{2}\left(\breve{h}_{p}\right)=\breve{h}_{p}$ (naturally, it is $F^{2}(0)=0, F^{2}(\hat{b})=\hat{b}$ and $F^{2}(\infty)=0$ ). Taking the derivative of (A7) yields

$$
F^{2^{\prime}}\left(b_{t}\right)=x\left(b_{t}\right) F^{\prime}\left(b_{t}\right) \text {, }
$$

where 


$$
x\left(h_{t}\right)=\frac{\varphi \omega\left(F\left(h_{t}\right)\right)^{\psi+\delta-1}}{1+\omega\left(F\left(h_{t}\right)\right)^{\delta}}\left[\psi+\delta\left(1-\frac{\omega\left(F\left(h_{t}\right)\right)^{\delta}}{1+\omega\left(F\left(h_{t}\right)\right)^{\delta}}\right)\right] .
$$

We can use $F(0)=0$ and $F^{\prime}(0)=\infty$ with (A8) and (A9) to verify that $F^{2^{\prime}}(0)=\infty$. Furthermore, using (A9), it is straightforward to establish that

$$
x\left(b_{t}\right)\left\{\begin{array}{lll}
>0 & \text { if } & F\left(b_{t}\right)<\xi \\
<0 & \text { if } & F\left(b_{t}\right)>\xi
\end{array} .\right.
$$

Nevertheless, the properties of the function $F\left(b_{t}\right)$ reveal that there are two points $k_{1}$ and $k_{1}$, such that $k_{1}<\xi<k_{2}$, for which $F(\bullet)=\xi$. Therefore, $x\left(b_{t}\right)>0$ for $h_{t} \in\left[0, k_{1}\right)$ and $h_{t} \in\left(k_{2}, \infty\right)$, while $x\left(h_{t}\right)<0$ for $h_{t} \in\left(k_{1}, k_{2}\right)$. Together with (A6) and (A8), these results imply that that

$$
F^{2^{\prime}}\left(h_{t}\right)\left\{\begin{array}{lll}
>0 & \text { if } & h_{t} \in\left[0, k_{1}\right) \\
<0 & \text { if } & h_{t} \in\left(k_{1}, \xi\right) \\
>0 & \text { if } & h_{t} \in\left(\xi, k_{2}\right) \\
<0 & \text { if } & h_{t} \in\left(k_{2}, \infty\right)
\end{array} .\right.
$$

Now, we want to consider what happens when $F^{\prime}(\hat{b})<-1$. Taking account that $F(\hat{b})=\hat{b}$, we can combine (A3), (A8) and (A9) to see that $F^{2^{\prime}}(\hat{b})=\left(F^{\prime}(\hat{b})\right)^{2}$, therefore $F^{\prime}(\hat{b})<-1 \Leftrightarrow F^{2 \prime}(\hat{b})>1$. Together with $F^{2}(+\infty)=0$ and (A11), this result implies that there must be a point, say $\breve{h}_{2}>k_{2}>\hat{b}$ such that $F^{2}\left(\breve{h}_{2}\right)=\breve{h}_{2}$. In addition, together with $F^{2^{\prime}}(0)=+\infty$ and (A11) it also implies that there must be a point $\breve{h}_{1}<\xi<\hat{b}$ such that $F^{2}\left(\breve{h}_{1}\right)=\breve{h}_{1}$. Thus, the points $\left\{\breve{h}_{1}, \breve{h}_{2}\right\}$, such that $\breve{h}_{1}<\hat{b}<\breve{h}_{2}$, establish a period-2 cycle. Of course, for such a cycle to be a stable periodic equilibrium, $F^{\prime}(\hat{b})<-1$ is a necessary condition. If $-1<F^{\prime}(\hat{b})<0$ then $\hat{b}$ is asymptotically stable and a stable periodic equilibrium cannot exist. 


\section{References}

1. Acemoglu, D. 2009. Introduction to Modern Economic Growth, Princeton University Press.

2. Azariadis, C., and Drazen, A. 1990. "Threshold externalities in economic development," Quarterly Journal of Economics, 105, 501-526.

3. Azariadis, C., and Reichlin, P. 1996. "Increasing returns and crowding out," Journal of Economic Dynamics and Control, 20, 847-877.

4. Banerji, S., Bhattacharya, J., and Long, N.V. 2004. "Can financial intermediation induce endogenous fluctuations?" Journal of Economic Dynamics and Control, 28, 22152238.

5. Barro, R.J. 2001. "Human capital and growth," American Economic Review, 91, 12-17.

6. Bedard, K., and Herman, D.A. 2008. "Who goes to graduate/professional school? The importance of economic fluctuations, undergraduate field, and ability," Economics of Education Review, 27, 197-210.

7. Benhabib, J., and Farmer, R.E.A. 1994. "Indeterminacy and increasing returns," Journal of Economic Theory, 63, 19-41.

8. Benhabib, J., and Spiegel, M.M. 1994. "The role of human capital in economic development: evidence from aggregate cross-country data," Journal of Monetary Economics, 34, 143-173.

9. Betts, J.R., and McFarland, L.L. 1995. "Safe port in a storm: the impact of labour market conditions on community college enrolments," Journal of Human Resources, 30, $741-765$.

10. Bhattacharya, J., and Qiao, X. 2007. "Public and private expenditures on health in a growth model," Journal of Economic Dynamics and Control, 31, 2519-2535.

11. Bunzel, H. 2006. "Habit persistence, money, and overlapping generations," Journal of Economic Dynamics and Control, 30, 2425-2445.

12. de la Croix D., and Monfort, P. 2000. "Regional convergence and education funding," Journal of Population Economics, 13, 403-424.

13. DeJong, D.N., and Ingram, B.F. 2001. "The cyclical behaviour of skill acquisition," Review of Economic Dynamics, 4, 536-561.

14. Dellas, H., and Sakellaris, P. 2003. "On the cyclicality of schooling: theory and evidence," Oxford Economic Papers, 55, 148-172. 
15. Fanti, L., and Gori, L. 2011. "Public health spending, old-age productivity, and economic growth: chaotic cycles under perfect foresight," Journal of Economic Behavior and Organization, 78, 163-186.

16. Farmer, R.E.A. 1986. "Deficits and cycles," Journal of Economic Theory, 40, 77-88.

17. Glomm, G., and Ravikumar, B. 1992. "Public versus private investment in human capital: endogenous growth and income inequality," Journal of Political Economy, 100, 818-834.

18. Grandmont, J.M. 1985. "On endogenous competitive business cycles," Econometrica, 53, 995-1045

19. Gruber, J. 2006. "A tax-based estimate of the elasticity of intertemporal substitution," NBER working paper no. 11945

20. Johnson, M.T. 2013. “The impact of business cycle fluctuations on graduate school enrolment," Economics of Education Review, 34, 122-134.

21. Kaas, L., and Zink, S. 2007. "Human capital and growth cycles," Economic Theory, 31, 19-33.

22. Kapoor, M., and Ravi, S. 2010. "Elasticity of intertemporal substitution in consumption $(\sigma)$ : empirical evidence from a natural experiment," unpublished manuscript.

23. Lahiri, A., and Puhakka, M. 1998. "Habit persistence in overlapping generations economies under pure exchange," Journal of Economic Theory, 78, 176-186

24. Li, T.Y., and Yorke, J.A. 1975. "Period three implies chaos," The American Mathematical Monthly, 82, 985-992.

25. Lucas, R.E. Jr. 1988. "On the mechanics of economic development," Journal of Monetary Economics, 22, 3-42.

26. Michener, R., and Ravikumar, B. 1998. "Chaotic dynamics in a cash-in-advance economy," Journal of Economic Dynamics and Control, 22, 1117-1137.

27. Mulligan, C. 2002. "Capital, interest, and aggregate intertemporal substitution," NBER working paper no. 9373.

28. Palivos, T., and Varvarigos, D. 2010. "Pollution abatement as a source of stabilisation and long-run growth," Department of Economics working paper no. 11/04, University of Leicester. 
29. Reichlin, P. 1986. "Equilibrium cycles in an overlapping generations model with production," Journal of Economic Theory, 40, 89-102.

30. Reichlin, P. 1997. "Endogenous cycles in competitive models: an overview," Studies in Nonlinear Dynamics and Econometrics, 1, 175-185.

31. Rillaers, A. 2001. "Education and income inequality: The role of a social protection system," Journal of Population Economics, 14, 425-443.

32. Sakellaris, P., and Spilimbergo, A. 2000. "Business cycles and investment in human capital: international evidence on higher education," Carnegie-Rochester Conference Series on Public Policy, 52, 221-256.

33. Seegmuller, T., and Verchère, A. 2004. "Pollution as a source of endogenous fluctuations and periodic welfare inequality in OLG economies," Economics Letters, 84, 363-369. 